\title{
Vitamin D Deficiency is Associated with Type 2 Diabetes Mellitus in HIV Infection
}

\author{
Zsofia SZEP, MD, MSCE ${ }^{1,2,3}$, Giovanni GUARALDI, MD ${ }^{4}$, Samir S. SHAH, MD, MSCE ${ }^{1,2,5}$, LO \\ RE Vincent 3rd, MD, MSCE ${ }^{1,2,3}$, Sarah J RATCLIFFE, PhD ${ }^{1,3}$, Gabriella ORLANDO, MD ${ }^{4}$, \\ Frederica CARLI, MD ${ }^{4}$, Rosario ROSSI, MD ${ }^{4}$, Vincenzo ROCHIRA, MD ${ }^{4}$, and Pablo TEBAS, \\ $\mathrm{MD}^{2}$ \\ ${ }^{1}$ Center for Clinical Epidemiology and Biostatistics, University of Pennsylvania School of \\ Medicine, PA, USA \\ ${ }^{2}$ Department of Medicine, University of Pennsylvania School of Medicine, PA, USA \\ ${ }^{3}$ Department of Biostatistics and Epidemiology, University of Pennsylvania School of Medicine, \\ PA, USA \\ ${ }^{4}$ Department of Internal Medicine and Medical Specialties, University of Modena and Reggio \\ Emilia, Modena, Italy \\ ${ }^{5}$ Department of Pediatrics, University of Pennsylvania School of Medicine, and Division of \\ Infectious Diseases, The Children's Hospital of Philadelphia, Philadelphia, PA, USA
}

\section{Abstract}

Background-Metabolic complications, including type 2 diabetes mellitus (DM) and metabolic syndrome, are increasingly recognized among HIV-infected individuals. Low vitamin D levels increase the risk of type $2 \mathrm{DM}$, and vitamin $\mathrm{D}$ supplementation has been shown to decrease the risk of type $2 \mathrm{DM}$ in patients without HIV infection.

Objectives-The primary objective was to determine whether vitamin D deficiency (serum 25hyrdoxyvitamin $\mathrm{D}<20 \mathrm{ng} / \mathrm{mL}$ ) was associated with type $2 \mathrm{DM}$ among HIV-infected patients. Our secondary objective was to determine whether vitamin D deficiency was associated with metabolic syndrome in HIV.

\begin{abstract}
Methods-We conducted a cross-sectional study among subjects enrolled in the prospective Modena (Italy) HIV Metabolic Clinic Cohort. Clinical and laboratory data, including history of type $2 \mathrm{DM}$, fasting blood glucose, components of metabolic syndrome, and 25-hydroxyvitamin D levels, were obtained for all subjects.

Results-After adjusting for vitamin D supplementation, sex, age, body mass index, and hepatitis $\mathrm{C}$ virus co-infection, vitamin D deficiency was associated with type 2 DM (adjusted OR, $1.85 ; 95 \% \mathrm{CI}, 1.03-3.32 ; \mathrm{p}=.038$ ). The association between vitamin $\mathrm{D}$ deficiency and metabolic syndrome was not significant after adjusting for vitamin $\mathrm{D}$ supplementation, sex age and body mass index (adjusted OR 1.32; 95\% CI, 1.00-1.75; $\mathrm{p}=.053$ ).
\end{abstract}

Conclusions-Our study demonstrates an association between vitamin D deficiency and type 2 DM. Clinical trials are needed to better characterize the association between vitamin D deficiency

Address Correspondence to: Zsofia Szep Telephone: 215-500-5550, Fax: 267-639-6269, zsofia.szep@uphs.upenn.edu. Data presented previously and published as an abstract at $10^{\text {th }}$ International Workshop on Adverse Drug Reactions and Lipodystrophy in HIV. London, UK November 2008 
and type 2 DM in HIV infection and to evaluate whether vitamin D is able to prevent or delay the onset of type $2 \mathrm{DM}$.

\section{Keywords}

vitamin D deficiency; type 2 DM; insulin resistance; metabolic syndrome

\section{INTRODUCTION}

Patients infected with HIV are surviving longer because of antiretroviral therapy (ART). Consequently, there has been an increased awareness of adverse events related to ART, particularly metabolic complications such as insulin resistance [1,2]. The etiology of insulin resistance includes patient-level factors, HIV infection, certain protease inhibitors [3], and thymidine analogues [4]. Insulin resistance contributes to the development of type 2 diabetes mellitus (DM) and cardiovascular disease among HIV-infected patients.

Deficiency of vitamin D, the major steroid hormone involved in calcium homeostasis and bone metabolism, has been associated with impaired $\beta$-cell function and insulin resistance in humans [5, 6]. In HIV-uninfected patients, vitamin D deficiency (25-hydroxyvitamin D<20 $\mathrm{ng} / \mathrm{mL}$ ) is a risk factor for both type $2 \mathrm{DM}$ and metabolic syndrome [7-9]. However, in the setting of HIV infection, the clinical consequences of vitamin D deficiency, which has been reported to have a prevalence ranging from 12 to $86 \%$ [10-16], remain unclear. In particular, the association between vitamin D deficiency and type $2 \mathrm{DM}$ and metabolic syndrome among HIV-infected patients is not known.

Our primary objective was to determine whether a low serum concentration of 25hydroxyvitamin D was associated with type $2 \mathrm{DM}$ among HIV-infected patients. We hypothesized that a low 25-hydroxyvitamin $\mathrm{D}$ level was an independent risk factor for type 2 DM in HIV. Our secondary objective was to determine if vitamin D deficiency among HIV-infected patients was associated with the metabolic syndrome, for which the underlying mechanism is considered to be insulin resistance. We hypothesized that individuals with HIV and low serum concentrations of 25-hydroxyvitamin D would have metabolic syndrome which has been linked to higher cardiovascular risk, in comparison to individuals with higher serum concentrations of 25 hydroxyvitamin D levels. Confirmation of these associations would identify hypovitaminosis $\mathrm{D}$ as a potentially modifiable cardiovascular risk factor among HIV-infected patients.

\section{METHODS}

\section{Study Design and Participants}

We performed a cross-sectional study among subjects enrolled in the Modena HIV Metabolic Clinic Cohort, a prospective cohort study initiated in September 2004 to evaluate metabolic alterations among HIV-infected patients followed at the Metabolic Clinic of the University of Modena and Reggio Emilia School of Medicine (Modena, Italy) [17]. HIV infected patients with metabolic complications are referred to this clinic for further evaluation. Subjects have laboratory-confirmed HIV, provide informed consent, and complete a standardized questionnaire that collects demographic, medical, and HIV data at enrollment. Laboratory evaluation at enrollment includes blood for fasting lipids, fasting glucose, 25-hydroxyvitamin D, HIV RNA, immune function, and hepatitis status.

All subjects enrolled in the cohort between January 1, 2005 and January 31, 2008 who had documented 25-hydroxyvitamin D level were eligible for inclusion. For our primary objective, we included subjects with a 25-hydroxyvitamin D level and either fasting serum 
glucose level or documented type $2 \mathrm{DM}$ diagnosis and use of insulin or oral antidiabetic medication. For our secondary objective, we included subjects with a 25-hydroxyvitamin D level and presence or absence of the metabolic syndrome diagnosis. This study was reviewed and approved by the Institutional Review Boards of the University of Modena and University of Pennsylvania.

\section{Main Study Outcomes}

The primary outcome was type $2 \mathrm{DM}$, defined as a fasting glucose $\geq 126 \mathrm{mg} / \mathrm{dL}$ [18], a physician's diagnosis of type $2 \mathrm{DM}$, and/or receipt of an oral anti-diabetic agent or insulin.

As a secondary outcome, we evaluated the metabolic syndrome, defined by the presence of any 3 of the following 5 characteristics according to the National Cholesterol Education Program's Adult Treatment Panel III report: abdominal obesity based on waist circumference ( $>102 \mathrm{~cm}$ in men and $>88 \mathrm{~cm}$ in women), triglycerides $\geq 150 \mathrm{mg} / \mathrm{dL}$, high density lipoprotein (HDL) $<40 \mathrm{mg} / \mathrm{dL}$ for men and $<50 \mathrm{mg} / \mathrm{dL}$ for women, blood pressure $\geq$ $130 / \geq 85 \mathrm{~mm} \mathrm{Hg}$, and fasting glucose $\geq 110 \mathrm{mg} / \mathrm{dL}$ [19].

\section{Data Collection}

Demographic (age; sex), clinical (height; body weight; blood pressure; history of type 2 DM [as defined above]), and HIV data (duration of HIV diagnosis; antiretroviral use and duration) were recorded at subject enrollment.

Blood was drawn from all subjects after at least 8 hours of fasting for determination of HIV RNA (Abbott RealTime HIV-1 assay; Abbott Laboratories; lower limit of detection: 50 copies/mL); CD4 T lymphocyte count; hepatitis C virus (HCV) antibody (anti-HCV; Abbott HCV EIA 3.0 enzyme immunoassay; Abbott Laboratories); glucose (Rapidpoint 400 analyzer; Bayer); total cholesterol (Cholesterol/HP; Roche); triglyceride (Triglycerides/ GPO; Roche); HDL (HDL-C plus 3rd generation; Roche); and 25-hydroxyvitamin D (DiaSorin 25-hydroxyvitamin D chemiluminescence immunoassay; Stillwater, MN). Vitamin D deficiency was defined as a serum 25 -hydroxyvitamin D level $<20 \mathrm{ng} / \mathrm{mL}$ ( 50 $\mathrm{nmol}$ per liter). Vitamin D insufficiency was defined as 25 -hydroxyvitamin D $330 \mathrm{ng} / \mathrm{mL}$ and $\geq 20 \mathrm{ng} / \mathrm{ml}$. [20]

\section{Statistical Analysis}

Differences between groups were assessed using Student's t-tests for continuous variables and chi-square tests for categorical variables. Multivariable logistic regression determined unadjusted and adjusted odds ratios (ORs) and 95\% confidence intervals (CIs) of type 2 DM and metabolic syndrome between subjects with and without low vitamin D. Potential confounding variables evaluated included age, sex, body mass index, vitamin D supplementation, season of vitamin D measurement, thymidine analogues, specific protease inhibitors such as lopinavir, and HCV co-infection.

Backward elimination procedures removed non-significant potential confounders from adjusted models. All analyses were performed using STATA 10.0 (College Station, TX).

\section{RESULTS}

\section{Vitamin D Status}

The mean serum concentration of 25-hydroxyvitamin D was $19 \mathrm{ng} / \mathrm{mL}$ (95\% Confidence Interval $[\mathrm{CI}]=18-20 \mathrm{ng} / \mathrm{mL}$ ). A total of $64 \%$ were vitamin D deficient (25-hydroxyvtitamin $\mathrm{D}<20 \mathrm{ng} / \mathrm{mL}$ ), and an additional $20 \%$ of the subjects had vitamin D insufficiency. Among 
1405 subjects with available information regarding supplementation, 93 (7\%) were taking a vitamin D supplement (7500 IUs vitamin D3 weekly).

\section{Association Between 25(OH) Vit D Levels and Type 2 DM}

Mean 25-hydroxyvitamin D levels among type 2 DM subjects were lower than in subjects without type 2 DM (Table 1). After adjusting for vitamin D supplementation, sex, age, body mass index and HCV co-infection, vitamin D deficiency was associated with type $2 \mathrm{DM}$ (adjusted OR, 1.85; 95\% CI, 1.03-3.32; p=.038) (Table 2).

\section{Association Between 25-hydroxyvitamin D Levels and Metabolic Syndrome}

Overall among 1687 subjects, 412 (24\%) met the definition of metabolic syndrome. Mean 25-hydroxyvitamin D levels among subjects with metabolic syndrome were lower than in subjects without metabolic syndrome $(17[ \pm 12]$ versus $20[ \pm 10] \mathrm{ng} / \mathrm{mL} ; \mathrm{p}<.001)$. The association between vitamin $\mathrm{D}$ deficiency was not significant after adjusting for age, sex, body mass index, and vitamin D supplementation (Table 2).

\section{DISCUSSION}

Our study identified a high prevalence of vitamin D deficiency among HIV-infected patients. Patients with type $2 \mathrm{DM}$ in this study had lower 25-hydroxyvitamin D levels compared with subjects without type $2 \mathrm{DM}$, although both groups met criteria for vitamin D deficiency. We also demonstrated that vitamin D deficiency was independently associated with type $2 \mathrm{DM}$ in this population. However, we did not find an association between vitamin D deficiency and metabolic syndrome.

The prevalence of vitamin D deficiency among the HIV-infected patients in this sample was similar to that reported by Garcia-Aperico et al [11] among 30 HIV-infected males in Spain $(86 \%)$. The prevalence of vitamin D deficiency in our population was higher than in several previous studies, which reported prevalences of $12 \%$ to $47 \%$ [12-16]. In contrast, a large Italian study of more than 6000 outpatients and inpatients reported a 52\% prevalence of vitamin D deficiency [21]. The higher prevalence of vitamin D deficiency in this study may be related to the northern geographic location of Modena $\left(44^{\circ} \mathrm{N}\right)$ which may lead to more indoor activities and less sun exposure.

Several cross-sectional studies have shown that low serum 25-hydroxyvitamin D concentration is associated with impaired glucose tolerance in HIV-uninfected patients $[7,8]$. In the Nurses' Health Study, Pittas et al [8] demonstrated an inverse association between vitamin D supplementation and the development of type 2 DM. A combined daily intake of at least $1,200 \mathrm{mg}$ of calcium and $800 \mathrm{IUs}$ of vitamin D was associated with a $33 \%$ lower risk of type $2 \mathrm{DM}$. The findings of our study are consistent with those of HIV-uninfected individuals and suggest that the association between vitamin D deficiency and type $2 \mathrm{DM}$ is also present among HIV- infected individuals.

In our study, subjects with metabolic syndrome had lower serum 25-hydroxyvitamin D levels. However, we did not find an association between vitamin D deficiency and metabolic syndrome.

The role of HIV and ART in vitamin D deficiency is complex. HIV might affect $a-$ hydroxylation of 25-hydroxyvitamin $\mathrm{D}$ in the kidneys to its active form 1, 25dihydroxyvitamin D [22]. Protease inhibitors and non-nucleoside reverse transcriptase inhibitors utilize the cytochrome p450 enzyme, which is also the site of hydroxylation of vitamin D. Thus, certain antiretrovirals may also predispose to vitamin D deficiency [23]. If individuals with HIV are at a higher risk of developing vitamin D deficiency due to HIV and 
ART, then the consequences of vitamin D deficiency, such as type $2 \mathrm{DM}$, may be more prevalent among HIV-infected individuals, as shown by our results.

Additionally, inflammation has been shown to play a key role in the development of type 2 DM and metabolic syndrome. Vitamin D has anti-inflammatory effects among HIVuninfected individuals, which may explain its protective role in type $2 \mathrm{DM}$ and cardiovascular disease [24,25]. Vitamin D may lower the risk of type 2 DM and metabolic syndrome in HIV by reducing the heightened inflammation associated with HIV infection . [26]

Our study had several limitations. First, since we used a cross-sectional design, we cannot establish whether the relationship between vitamin D deficiency and type $2 \mathrm{DM}$ is causal. $A$ small prospective study by van Den Bout et al evaluated the impact of vitamin $D$ supplementation on insulin sensitivity in 20 HIV infected subjects. At 24 weeks insulin sensitivity was decreased but by 48 weeks insulin sensitivity returned to baseline [27 ]. Further larger prospective studies are needed to evaluate whether vitamin D deficiency may be a modifiable risk factor for the development of type 2 DM.

Second, our definition of type 2 DM may have resulted in outcome misclassification. Patients with type $2 \mathrm{DM}$ with well controlled fasting blood glucose levels who failed to report anti-diabetic medication use may have been misclassified as not having type 2 DM. However, such outcome misclassification would have caused us to underestimate the association between vitamin D deficiency and type $2 \mathrm{DM}$.

Additionally, it is also possible that vitamin D deficiency is associated with insulin resistance, even among those not meeting criteria for type 2 DM. Patients with more modest degrees of insulin resistance were classified as not having type 2 DM in this study. Such misclassification would bias our results towards the null. This limitation would cause us to underestimate the association between vitamin D deficiency and insulin resistance.

Lastly, subjects were from Italy, a homogeneous population, potentially limiting the generalizability of our results.

In summary, we demonstrated that vitamin D deficiency was independently associated with type 2 DM in HIV. Future studies should examine whether vitamin D supplementation can prevent or treat type 2 DM in HIV and possibly reduce complications associated with HIV infection and its treatment.

\section{Acknowledgments}

Dr. Szep received support from the National Institute of Allergy and Infectious Diseases (T32 AI055435-06) and Penn Center for AIDS Research (P30 AI 045008). Dr. Shah received support from the National Institute of Allergy and Infectious Diseases (K01 AI73729) and the Robert Wood Johnson Foundation under its Physician Faculty Scholar Program. Dr. Lo Re received support from the National Institute of Allergy and Infectious Diseases (K01 AI07001). The content is solely the responsibility of the authors and does not necessarily represent the official views of the National Institutes of Health or the Robert Wood Johnson Foundation.

\section{References}

1. De Wit S, Sabin CA, Weber R, Worm SW, Reiss P, Cazanave C, et al. Data Collection on Adverse Events of Anti-HIV Drugs (D:A:D) study. Incidence and risk factors for new-onset diabetes in HIVinfected patients: the Data Collection on Adverse Events of Anti-HIV Drugs (D:A:D) study.

Diabetes Care. 2008; 31(6):1224-1229. [PubMed: 18268071] 
2. Tien PC, Schneider MF, Cole SR, Levine AM, Cohen M, DeHovitz J, et al. Antiretroviral therapy exposure and incidence of diabetes mellitus in the Women's Interagency HIV Study. AIDS. 2007; 21(13):1739-1745. [PubMed: 17690572]

3. Koster JC, Remedi MS, Qiu H, Nichols CG, Hruz PW. HIV protease inhibitors acutely impair glucose-stimulated insulin release. Diabetes. 2003; 52(7):1695-1700. [PubMed: 12829635]

4. Brown TT, Li X, Cole SR, Kingsley LA, Palella FJ, Riddler SA, et al. Cumulative exposure to nucleoside analogue reverse transcriptase inhibitors is associated with insulin resistance markers in the Multicenter AIDS Cohort Study. AIDS. 2005; 19(13):1375-1383. [PubMed: 16103768]

5. Pittas AG, Lau J, Hu FB, Dawson-Hughes B. The role of vitamin D and calcium in type 2 diabetes. A systematic review and meta-analysis. J Clin Endocrinol Metab. 2007; 92(6):2017-2029. [PubMed: 17389701]

6. Chiu KC, Chu A, Go VL, Saad MF. Hypovitaminosis D is associated with insulin resistance and beta cell dysfunction. Am J Clin Nutr. 2004; 79(5):820-825. [PubMed: 15113720]

7. Mattila C, Knekt P, Mannisto S, Rissanen H, Laaksonen MA, Montonen J, et al. Serum 25hydroxyvitamin D concentration and subsequent risk of type 2 diabetes. Diabetes Care. 2007; 30(10):2569-2570. [PubMed: 17626891]

8. Pittas AG, Dawson-Hughes B, Li T, Van Dam RM, Willett WC, Manson JE, et al. Vitamin D and calcium intake in relation to type 2 diabetes in women. Diabetes Care. 2006; 29(3):650-656. [PubMed: 16505521]

9. Ford ES, Ajani UA, McGuire LC, Liu S. Concentrations of serum vitamin D and the metabolic syndrome among U S adults. Diabetes Care. 2005; 28(5):1228-1230. [PubMed: 15855599]

10. Stephensen CB, Marquis GS, Kruzich LA, Douglas SD, Aldrovandi GM, Wilson CM. Vitamin D status in adolescents and young adults with HIV infection. Am J Clin Nutr. 2006; 83(5):11351141. [PubMed: 16685057]

11. Garcia Aparicio AM, Munoz Fernandez S, Gonzalez J, Arribas JR, Pena JM, Vazquez JJ, et al. Abnormalities in the bone mineral metabolism in HIV-infected patients. Clin Rheumatol. 2006; 25(4):537-539. [PubMed: 16208429]

12. Van Den Bout-Van Den Beukel CJ, Fievez L, Michels M, Sweep FC, Hermus AR, Bosch ME, et al. Vitamin D deficiency among HIV type 1-infected individuals in the Netherlands: effects of antiretroviral therapy. AIDS Res Hum Retroviruses. 2008; 24(11):1375-1382. [PubMed: 18928396]

13. Lattuada E, Lanzafame M, Zoppini G, Concia E, Vento S. No influence of nevirapine on vitamin D deficiency in HIV-infected patients. AIDS Res Hum Retroviruses. 2009; 25(8):849-850. [PubMed: 19618997]

14. Rodriguez M, Daniels B, Gunawardene S, Robbins GK. High frequency of vitamin D deficiency in ambulatory HIV-Positive patients. AIDS Res Hum Retroviruses. 2009; 25(1):9-14. [PubMed: 19108690]

15. Wasserman P, Rubin DS. Highly prevalent vitamin D deficiency and insufficiency in an urban cohort of HIV-infected men under care. AIDS Patient Care STDS. 2010; 24(4):223-227. [PubMed: 20377437]

16. Bang UC, Shakar SA, Hitz MF, Jespersen MS, Andersen O, Nielsen SD, et al. Deficiency of 25hydroxyvitamin D in male HIV-positive patients: a descriptive cross-sectional study. Scand J Infect Dis. 2010; 42(4):306-310. [PubMed: 20085419]

17. Guaraldi G, Orlando G, Squillace N, De Santis G, Pedone A, Spaggiari A, et al. Multidisciplinary approach to the treatment of metabolic and morphologic alterations of HIV-related lipodystrophy. HIV Clinical Trials. 2006; 7(3):97-106. [PubMed: 16880166]

18. American Diabetes A. Standards of medical care in diabetes--2008. Diabetes Care. 2008; 31(Suppl 1):S12-54. [PubMed: 18165335]

19. Grundy SM, Cleeman JI, Daniels SR, Donato KA, Eckel RH, Franklin BA, et al. American Heart Association, National Heart, Lung, and Blood Institute. Diagnosis and management of the metabolic syndrome: an American Heart Association/National Heart, Lung, and Blood Institute Scientific Statement.[erratum appears in Circulation. 2005; 112(17):e297]. Circulation. 2005; 112(17):2735-2752. [PubMed: 16157765] 
20. Holick MF. Vitamin D deficiency.[see comment]. N Engl J Med. 2007; 357(3):266-281. [PubMed: 17634462]

21. Cigolini M, Miconi V, Soffiati G, Fortanato A, Iagulli MP, Lombardi S, et al. Hypovitaminosis D among unselected medical inpatients and outpatients in Northern Italy. Clin Endocrinol(Oxf). 2006; 64(4):475. [PubMed: 16584525]

22. Villamor E. A potential role for vitamin D on HIV infection? Nutr. Rev. 2006; 64:226-233.

23. Cozzolino M, Vidal M, Arcidiacono MV, Tebas P, Yarasheski KE, Dusso AS. HIV-protease inhibitors impair vitamin D bioactivation to 1,25-dihydroxyvitamin D. AIDS. 2003; 17(4):513520. [PubMed: 12598771]

24. Petchey WG, Hickman IJ, Duncan E, Prins JB, Hawley CM, Johannes BH, et al. The role of 25hydroxyvitamin D deficiency in promoting insulin resistance and inflammation in patients with chronic kidney disease: a randomized controlled trial. BMC Nephrol. 2009; 10:41. [PubMed: 20003316]

25. Zitterman A, Firsch S, Berthold HK, Gottin C, Kuhn J, Kleesiek K, et al. Vitamin D supplementation enhances the beneficial effects of weight loss on cardiovascular disease risk markers. Am J Clin Nutr. 2009; 89(5):1321-1327. [PubMed: 19321573]

26. Michos ED, Melamed ML. Vitamin D and cardiovascular disease risk. Curr Opin Clin Nutr Metab Care. 2008; 11(1):7-12. [PubMed: 18090651]

27. van den Bout-van den Beukel CJ, Van den Bos M, Oyen WJ, Hermus AR, Sweep FC, Tack CJ, et al. The effect of cholecalciferol supplementation on vitamin D levels and insulin sensitivity is dose related in vitamin D deficient HIV-1-infected patients. HIV Med. 2008; 9(9):771-779. [PubMed: 18754805] 


\section{Table 2}

Factors associated with type 2 DM and Metabolic Syndrome

\begin{tabular}{|c|c|c|c|c|}
\hline Characteristics & $\begin{array}{c}\text { Adjusted Odds Ratio* }(95 \% \\
\text { Confidence Interval) } \\
\text { Type } 2 \text { DM }\end{array}$ & P Value & $\begin{array}{l}\text { Adjusted Odds Ratio }{ }^{*}(95 \% \\
\text { Confidence Interval) } \\
\text { Metabolic Syndrome }\end{array}$ & P Value \\
\hline 25-hydroxyvitamin $D<20 \mathrm{ng} / \mathrm{mL}$ & $1.85(1.03-3.32)$ & .038 & $1.32(1.00-1.75)$ & .053 \\
\hline Vitamin D Supplementation & $.87(.35-2.18)$ & .767 & $.76(.42-1.36)$ & .352 \\
\hline Gender (male) & $1.57(.89-2.82)$ & .128 & $1.05(.79-1.40)$ & .722 \\
\hline Age, per year & $1.08(1.05-1.11)$ & $<.01$ & $1.05(1.03-1.06)$ & $<.01$ \\
\hline BMI $\mathrm{kg} / \mathrm{m}^{2}$, per $1 \mathrm{U}$ & $1.09(1.03-1.14)$ & $<.01$ & $1.15(1.11-1.19)$ & $<.01$ \\
\hline Hepatitis $\mathbf{C}$ Virus coinfection ${ }^{\dagger}$ & $1.42(.86-2.36)$ & .171 & & \\
\hline
\end{tabular}

Abbreviations: BMI, Body Mass Index

Model adjusted for vitamin D supplementation, gender, age, body mass index,

${ }^{\dagger}$ Type 2 DM model adjusted for hepatitis C virus co-infection 